\title{
How flavour and appearance affect human feeding
}

\author{
By Barbara J. Rolls, E. A. Rowe and E. T. Rolls, University of Oxford,
} Department of Experimental Psychology, South Parks Road, Oxford $O X_{\mathrm{I}}{ }_{3} U D$

The amount of food eaten during a meal is determined by both internal physiological events and factors in the environment such as the availability, cost and presentation of food as well as the time of day and the social situation (Pliner, 1978; Rodin, I980). It is the purpose of this paper to consider the ways in which the different sensory aspects of food presentation such as flavour, appearance, portion size and shape influence food intake in human subjects within the range of normal body-weights. One way in which the sensory properties of food influence feeding is by contributing to a form of satiety which is partly specific for the particular food eaten.

\section{Sensory specific satiety}

Cabanac (197I) has suggested that the palatability or subjective pleasantness of a particular food depends on its usefulness as determined by internal bodily signals. He found that sweet solutions of sucrose and a food-related smell (orange) were rated as pleasant when subjects were hungry, but after they had been given a load of glucose or sucrose either orally or directly into the stomach, the previously pleasant taste or smell became less pleasant. We wished to know whether such changes in palatability were relevant to normal feeding and could be found with real foods after they had been consumed. Thus we determined the effect of eating one food to satiety on the subjective pleasantness of that food and of other foods which had not been eaten (Rolls, Rolls et al. 1981). Twenty-four subjects rated the taste of eight foods (cheese on cracker, sausage, chicken, walnuts, bread, raisins, banana, cookies) on a scale where +2 was very strong liking and -2 was very strong dislike. After this initial rating, subjects were given a plate of either cheese on crackers or sausages and instructed to eat as much as they liked. Two minutes after the end of the meal subjects rerated the tastes of the eight foods. It was found (see Fig. I) that the liking for the food eaten went down significantly more than for the foods not eaten $(P<0.01)$. The changes were relatively specific to the food which had been eaten. There was little change in the foods not eaten, except that after eating sausages the subjects' liking for chicken showed some decrease. This indicates that there may be interactions between foods of the same type, for example, meats. The fruits (raisins and bananas) and the cookies were liked slightly more after eating either sausages or cheese on crackers. This could be because people are accustomed to eating these foods as a last course or because there was a bigger contrast between these foods and the food eaten. We conclude that after a meal there is a decrease in the liking for the food eaten, with other foods remaining relatively unchanged. Furthermore, it was found that if the 


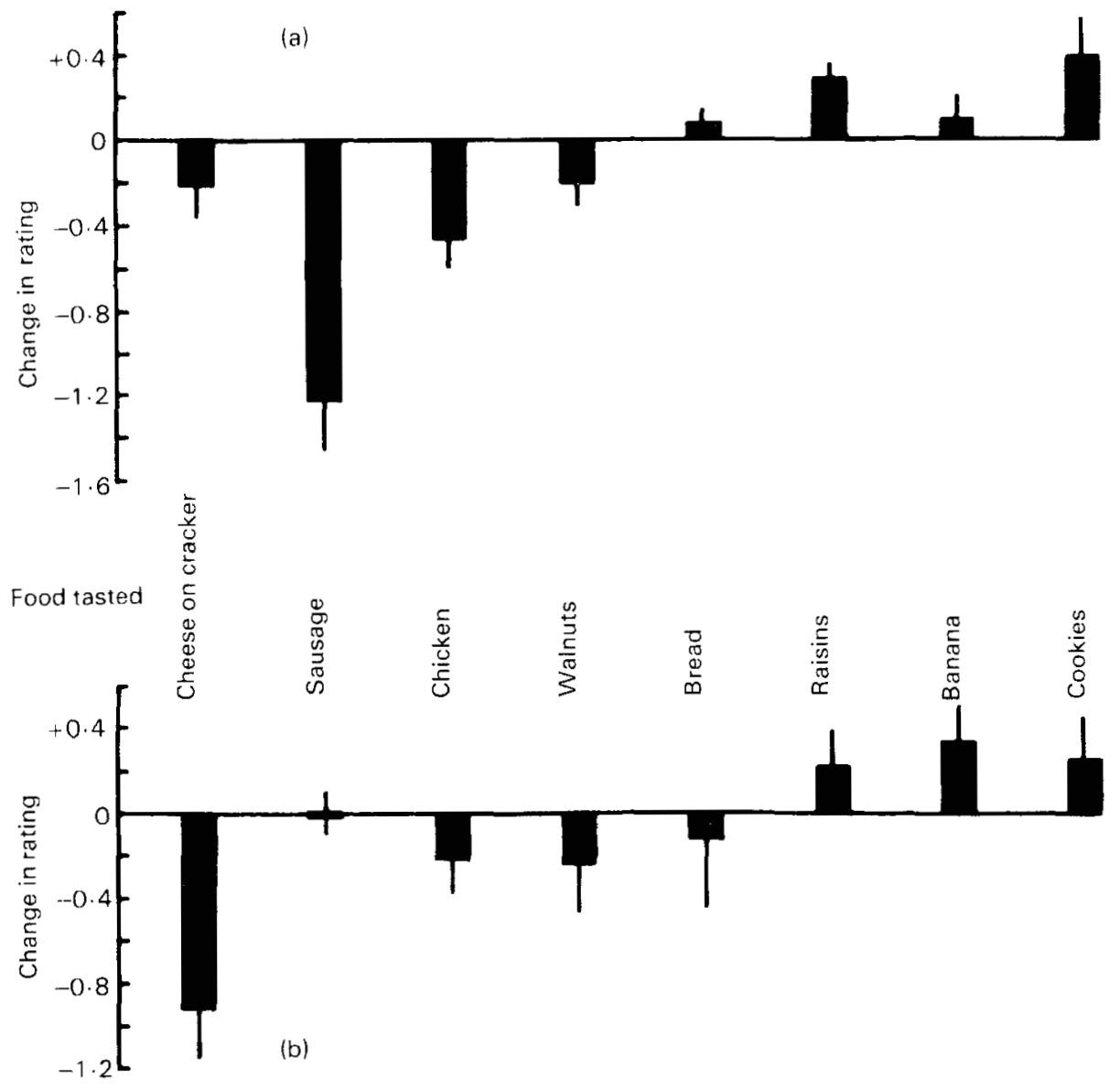

Fig. I. The mean ( $t$ SEM) change in ratings of liking from before the first course to 2 min after the first course, for the food eaten in the first course and seven other foods. The results are shown separately for (a) sausage and (b) cheese on cracker in the first course. The results show that changes in liking are relatively specific to the food which has been eaten.

subjects were given an unexpected second course of either the same food they had just consumed or another food, the changes in liking over the first course were correlated with the amount eaten in the second course $(P<0.001$ for the correlation between the change in liking and subsequent intake). Thus when the liking for a particular food is decreased because it has been eaten in a first course, relatively little of that food is eaten in a second course, but the intake of other foods which have not been eaten remains relatively high.

One factor which influences the pleasantness of the smell or taste of food appears to be the internal state or need for particular nutrients (Cabanac, i971). In support of this view Cabanac found that a decrease in pleasantness occurred only if the substance ingested was similar nutritionally to that tasted, i.e. glucose affected sucrose but not salt. This decrease in the pleasantness of sucrose solutions developed slowly after a glucose load, and maximal changes were seen about 
$45 \mathrm{~min}$ after the load. If sucrose was swilled in the mouth, but expectorated rather than swallowed, there was no change in the pleasantness of sweet solutions. Also, the pleasantness of the taste of sucrose decreased when glucose was tubed directly into the stomach or duodenum (Cabanac \& Fantino, 1977). Thus these results favour the post-ingestive consequences of a meal as an explanation for decreases in pleasantness. Other studies, however, favour the hypothesis that the sensory qualities of a meal play an important part in the change in palatability. Ingestion of the non-nutritive sweetener, cyclamate, has been found to be nearly as effective in reducing the pleasantness of the taste of sucrose solutions as is the ingestion of glucose (Wooley et al. 1972). Also, in the experiment described previously, in which one of eight foods tasted was ingested, we inferred that sensory or cognitive factors must be important because changes in liking were found just 2 min after the end of a meal and there were no further changes after 20 min (Rolls, Rolls $e t$ al. I98I). In addition, it was found that although there were some interactions between foods, the changes in liking were relatively specific to the particular food which had been eaten, so that it appeared likely that the satiety was related more to the sensory properties of each food rather than to the internal changes produced by the foods which were in some cases nutritionally similar (Rolls, Rolls et al. 1981). Furthermore, in other experiments we found specific changes in pleasantness when the substance ingested had little energy content, i.e. low-energy orange drink versus a meat extract flavoured drink. For these reasons, we have termed this phenomenon 'sensory specific satiety'. Because this indicates that there must be an important sensory component in the subjective response to food and the termination of eating, we have investigated the contribution of different sensory properties of foods such as flavour, colour and shape to satiety and palatability. First, we show how the changes in palatability which develop during a meal can affect food intake.

\section{Influence of variety on energy intake}

Since decreases in palatability occur for foods which have been consumed, more food is likely to be eaten of a varied than of a monotonous diet. We have tested this proposition in a series of experiments (Rolls, Rowe et al. $198 \mathrm{I}$ ). If normal weight subjects were offered in succession sandwiches with four different fillings for lunch, they ate a third more $(P<0.001)$ than if offered four successive plates of sandwiches all with the same filling. Also, if three flavours of yoghurt which differed in flavour, texture, and colour were offered in succession, significantly more $(P<0.01)$ was consumed than if one flavour was offered even if that single flavour was the favourite (also $P<0.01$ ).

Thus because satiety is partly specific to a particular food that has been eaten, offering a variety maintains appetite for a longer period than a monotonous meal. The sensory aspects of foods which could contribute to this specific component of satiety include the flavour, colour, texture, shape and temperature of foods. To determine to what extent these different sensory aspects of food can contribute to the development of sensory specific satiety and to the increase in food intake 
caused by variety, the following investigations were undertaken. We will consider the influence of flavour, and then a group of factors which all affect the appearance of food (i.e. colour, portion size and shape).

\section{Effect of flavour on energy intake}

The flavour of food is an important determinant of its acceptability. Some flavours are intrinsically unpleasant, but like coffee and chili peppers can become widely acceptable (Rozin, 1976). Flavour is not only important for determining whether or not a food will be accepted, but people can learn to use the tastes of foods as a guide to how much should be eaten, i.e. they learn that particular flavours are associated with a particular suppression of hunger (Booth, I98I). In previous studies on the specificity of satiety, the taste and odour of foods have been emphasized as contributory factors. For example, the non-nutritive sweetener, cyclamate, was found to be nearly as effective as glucose in reducing the pleasantness of sucrose (Wooley et al. 1972). It has also been reported that meal-related odours, but not non-food odours, were reduced in pleasantness after consumption of a meal (Duclaux et al. 1973). We have now run three different experiments in which we have assessed the contribution of changes in flavour to the enhancement of intake by variety.

Having found that intake was enhanced when yoghurts which differed in flavour, texture and appearance were presented in succession, we conducted a similar study in which three flavours of yoghurt were offered, but the texture and appearance were kept constant. Although the flavours (raspberry, strawberry, and cherry) were readily distinguishable there was no increase in intake in the variety condition compared with intake of just the favourite flavour (Rolls, Rowe et al. I $98 \mathrm{I})$.

In a second study on the effect of altering flavour only we offered school children orange, mint, or coffee flavoured chocolates (Matchmakers, Rowntree Mackintosh) which were of similar appearance and texture. Again, although the flavours were distinctive, there was no enhancement of intake when the succession of different flavours was offered compared with the intake of the individual flavours (B. J. Rolls, E. A. Rowe and E. T. Rolls, unpublished results).

It appears from these two studies that altering flavour alone does not lead to an enhancement of intake when a variety is offered. It is possible, however, that our particular choice of foods was masking an effect of variety. Although the foods were distinctive in flavour, they had strong background flavours coming from either the yoghurt or chocolate and all were sweet. Cabanac (1971) found that sweet tastes were reduced in pleasantness more than salty tastes after loads of sugar solutions. Thus we predicted that if foods differed fundamentally in flavour, an enhancement of intake would be found if a variety of such foods was presented in succession.

The main difficulty with a study in which flavour alone changes radically is to find foods that are palatable, of the same nutrient composition, and of similar appearance and texture. Our choice was cream cheese sandwiches flavoured with 
either salt, curry powder, or lemon essence and saccharin. Many potential subjects disliked one of the sandwich types and were thus not included since a refusal to eat a particular food masks the effects of variety. For the twenty-four subjects for whom the initial liking for the three flavours was similar there was a $15 \%$ enhancement of intake when the three flavours were presented in succession compared with the intake of the favourite flavour $(P<0.05)$ (B. J. Rolls, E. A. Rowe and E. T. Rolls, unpublished results). Thus changes in the flavours of foods presented in a meal can lead to an enhancement of total intake, but it appears that the contrast of flavour, if not accompanied by changes in appearance or texture, must be large before this enhancement is seen.

\section{Influence of the appearance of foods on energy intake}

The appearance of food is an important determinant of whether food will be ingested. It was interesting to discover a population of neurones in the lateral hypothalamus and substantia innominata of the hungry monkey which responded immediately before eating, that is, as soon as the monkey saw food, and while he looked at the food (Rolls et al. 1976, Rolls, 198I). The foods to which these neurones responded were the monkey's most preferred foods. During the course of testing it was found that the neuronal response decreased as the monkey became satiated. For example, at the beginning of a test the neurone would respond to the sight of a syringe containing $20 \%$ sucrose solution, but as the monkey drank this solution, this visual stimulus no longer affected the neurone and the monkey rejected the solution. However, after the monkey was apparently completely satiated as shown by rejection of the sucrose on $100 \%$ of the trials, the neurone responded strongly when the monkey was shown a peanut. Not only was the neurone responsive to the sight of this new food, but the monkey accepted the nut to eat (Rolls \& Rolls, 1982). This experiment showed that there is specificity of satiety at the neuronal level and led to our investigations of sensory specific satiety in man, and also emphasized the importance of visual mechanisms in the control of responses to food.

The electrophysiological evidence indicated that it would be worthwhile to investigate the effect of variation in the appearance of food on subjective responses to the food and on energy intake in man. In a study in which school children were offered Smarties (i.e. small discs of chocolate covered with a thin, coloured sugar layer) the only variable was the colour of the sweets. Subjects were tested three times, once with the favourite colour presented in four successive courses, with four different colours presented in succession, and with the four colours presented simultaneously in four successive courses. Subjects consumed the same amount in all three conditions, so the variety of colours used in this experiment was without effect on intake. In the condition in which just one colour had been presented the subjects had rated the pleasantness of all four colours before and after eating the single colour. It was found that the decrease in the pleasantness of the colour eaten was significantly $(P<0.01)$ greater for the colour eaten than for the colours not eaten (B. J. Rolls, E. A. Rowe and E. T. Rolls, unpublished results). Thus the taste 
of foods which differ only in colour is less appealing after they have been consumed than before eating. It seems likely that such subjective changes would affect the choice of foods to be consumed subsequently and would encourage switching between foods of different appearance.

Other types of studies have examined the effects of visual cues on food intake. Alterations in the salience of food by presenting it brightly or dimly lit (Ross, 1970), or in opaque or transparent wrapping (Johnson, 1974) had little effect on the total food intake of individuals of normal weight, although obese subjects were found to eat more of the more salient food. Thus several different experiments indicate that the total energy intake of individuals of normal weight is little affected by subtle changes in the appearance of food. However, visual cues related to the amount of food available are used to help determine the amount eaten. In a study in which subjects of normal weight consumed a liquid diet from either a visible or invisible food reservoir, intake varied markedly between the conditions, with $27 \%$ more being consumed when the reservoir was invisible and subjects could not visually monitor intake (Shaw, I973).

Thus the complete absence of food-related visual cues can affect food intake, but what is the effect of less extreme variation in the visual stimulus as may occur when the size of the portion offered is altered? Before discussing this type of experiment, it should be stressed that altering portion size not only influences the appearance of food, but may also affect the amount of food which is immediately available and thus influence the effort required to obtain more. Also, from past experience, subjects will have established views about the appropriateness of particular portion sizes. An early experiment indicated that the amount of food presented may not be an important influence on the amount eaten by individuals of normal weight. Subjects presented with either one sandwich at a time (with more available in a refrigerator) or three sandwiches together ate a mean of two sandwiches in both conditions (Nisbett, 1968 ). We have also examined the effect of varying the number of bite size sandwiches available at one time on the food intake of individuals of normal weight and have found no differences in food intake in different conditions (i.e. one half, six or twelve sandwiches each cut into eight pieces presented initially) (B. J. Rolls and E. A. Rowe, unpublished results). There have, however, been several studies which indicate that portion size may be an important determinant of meal size. Shaw (1973) found that subjects consumed more liquid diet (Metrecal) when it was freely available in 8 oz cups than when presented in 2 oz cups. Fuller (1980) working in Booth's laboratory found that increasing the size of portions of a variety of foods available in succession from a food dispenser increased energy intake significantly. Subjects were unaware of the changes in portion size, yet they showed some compensation by reducing the number of larger portions consumed. Thus portion size can be an important influence on the amount consumed during a meal. This could be because there is a tendency, particularly in obese individuals, to finish up food or clean the plate (Krassner et al. 1979). With the current emphasis on the variable energy requirements of individuals, innovative and responsible food producers ought to be 
concerned with offering the public a greater choice of portion sizes in supermarkets, restaurants and institutions.

Another way in which the appearance of food can vary is in its shape. Changes in shape also alter the feel of food in the mouth and it is known that food consistency and bite size determine the jaw movements during feeding (Thexton et al. 1980). It has been suggested that the muscle and neural activity associated
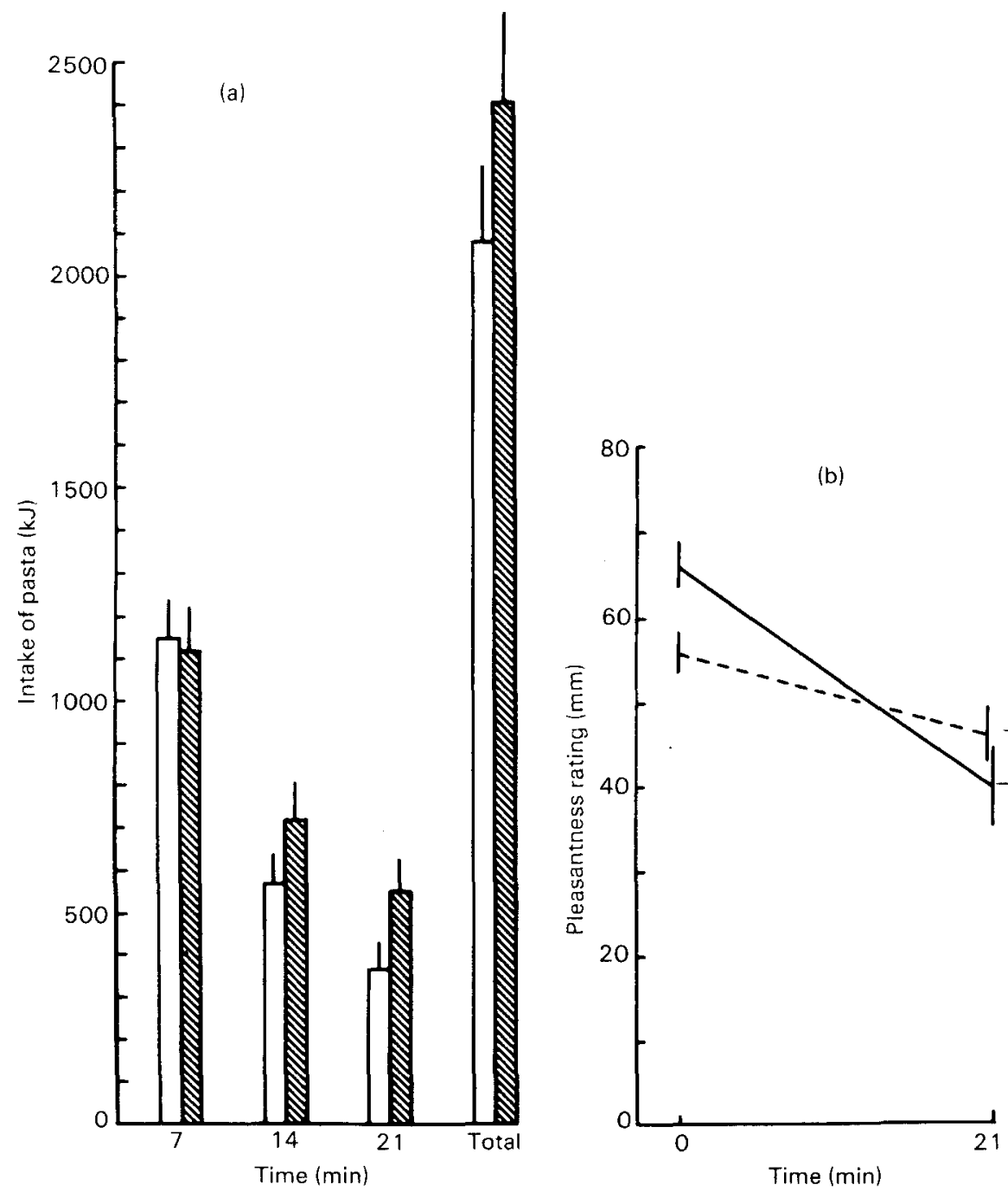

Fig. 2. (a) The mean ( $t \mathrm{SEM})$ amount of pasta eaten in three successive 7 min courses when subjects $\left(\begin{array}{ll}n & 24\end{array}\right)$ were given either three different shapes $(\mathbb{N})$ in succession or just the favourite shape ( $\square)$. The mean total intakes for the two conditions over the three courses is also shown. Significantly $(P<0.025)$ more pasta was consumed when the variety of shapes was offered. (b) The mean change in the pleasantness of the shape of pasta eaten (- - ) and the shapes not eaten (-- $)$ from before the meal to after the meal when just one shape was consumed during the test. There is a greater decrease in the pleasantness of the food consumed than of the foods not consumed. 
with mastication play an important role in food preference and may affect the amount of food eaten. The shape and size of food pellets offered to rats have been found to affect food intake (Tarozzi et al. 1980). To determine whether shape is also an important influence on feeding in humans, we assessed the effect of offering a variety of shapes of food on total energy intake in a meal. The foods used were different shapes of pasta served with equal portions of tomato sauce. Subjects were tested twice, once with just the favourite shape repeatedly presented, and once with three different shapes (spaghetti, bow ties and hoops) presented in three successive courses. We found (see Fig. 2 ) that there was a significant enhancement $(14 \%, P<0.025)$ of intake with the variety of shapes. In the condition in which just one shape was presented it was found that the pleasantness of the food eaten decreased more than that of the foods not eaten (see Fig. 2) and this could explain why the variety of shapes increased energy intake.

\section{Conclusions}

During the course of a meal the pleasantness of foods which have been eaten declines whereas the pleasantness of those foods not eaten remains relatively unchanged. Such changes in palatability affect the amounts of particular foods that will be eaten during the remainder of the meal. We have called this form of satiety 'sensory specific satiety'. Selective changes in the palatability of foods will favour switching from one food to another during a meal. Also, because satiety is relatively specific to foods which have been eaten, more is eaten of a varied meal than a monotonous one. The more different are foods, the greater will be the enhancement of intake by variety. The flavour and shape of foods can affect both the amount of food eaten and the subjective responses to foods. We have found that the successive presentation of foods which vary just in flavour or shape leads to a significantly greater intake in a meal than the presentation of just one flavour or shape. It is hoped that through this type of work a greater understanding of the factors in the environment which affect feeding will lead to potential means of gaining greater control of meal size in individuals who have difficulty in regulating body-weight.

The support of the Medical Research Council is gratefully acknowledged.

REFERENCES

Booth, D. A. (1981). In Brain, Behavior and Bodily Disease, p. I43 [H. Wiener, M. A. Hofer and A. J. Stunkard, editors]. New York: Raven Press.

Cabanac, M. (1971). Science, N.Y. 173, 1103.

Cabanac, M. \& Fantino, M. (1977). Physiol. Behav. 18, 1039.

Duclaux, R., Feisthauer, J. \& Cabanac, M. (1973). Physiol. Behav. 10, 1029.

Fuller, J. (I980). Human appetite and body size control. The roles of individual differences and food dependencies in human appetite and body size control processes. PhD Thesis, University of Birmingham, U.K.

Johnson, W. (I974). F. Pers. Soc. Psychol. 29, 843.

Krassner, H. A., Brownell, K. D. \& Stunkard, A. J. (1979). Behav. Res. Therapy 17, 155.

Nisbett, R. E. (1968). Science, N.Y. 159, I 254. 
Pliner, P. (1978). In Advances in Modern Nutrition, vol. 2, Diabetes, Obesity and Vascular Disease, p. $55^{I}$ [H. M. Katzen and R. J. Mahler, editors]. Washington: Hemisphere Publishing Corp.

Rodin, J. (1980). Int. J. Obesity 4,364 .

Rolls, B. J., Rolls, E. T., Rowe, E. A. \& Sweeney, K. (I981). Physiol. Behav. 27, I37.

Rolls, B. J., Rowe, E. A., Rolls, E. T., Kingston, B., Megson, A. \& Gunary, R. (1981). Physiol. Behav. 26, 2 I 5 .

Rolls, E. T. (1981). Br. Med. Bull. 37, 127.

Rolls, E. T., Burton, M. J. \& Mora, F. (1976). Brain Res. III, 53.

Rolls, E. T. \& Rolls, B. J. (1982). In Psychobiology of Human Food Selection [L. M. Barker, editor]. Westport, Connecticut: A.V.I. Publishing Co.

Ross, L. D. (1970). Cue- and cognition-controlled eating among obese and normal subjects. PhD Thesis, Columbia University.

Rozin, P. (1976). In Appetite and Food Intake, p. 285 [T. Silverstone, editor]. Berlin: Dahlem Konferenzen.

Shaw, J. (1973). The influence of type of food and method of presentation on human eating behaviour. PhD Thesis, University of Pennsylvania.

Tarozzi, G., Di Bella, L., Scalera, G. \& Rossi, M. T. (1980). In Proceedings of the Seventh International Conference on the Physiology of Food and Fluid Intake, Warsaw.

Thexton, A. J., Hiiemae, K. M. \& Crompton, A. W. (1980). F. Neurophysiol. 44, 456 .

Wooley, O. W., Wooley, S. C. \& Dunham, R. B. (1972). Physiol. Behav. 9, 765. 